H. Umemura

Nagoya Math. J.

Vol. 79 (1980), 47-67

\title{
SUR LES SOUS-GROUPES ALGEBRIQUES PRIMITIFS DU GROUPE DE CREMONA A TROIS VARIABLES
}

\author{
HIROSHI UMEMURA
}

$\mathrm{Au} 19 \mathrm{e}$ siècle le terme de géométrie algébrique désignait exclusivement la géométrie birationnelle. Aujourd'hui on n'accepterait pas cette définition, parce que l'objet de la géométrie algébrique est celui des invariants biréguliers plutôt que birationnels. Quelle que soit la définition adoptée, il est intéressant de connaître la structure de groupes des automorphismes birationnels. Mais celle-ci est très peu connue (Mumford [10]). La question suivante a été posée depuis longtemps; déterminer à conjugaison près tous les sous-groupes algébriques connexes et maximaux du groupe de Cremona $C r_{n}$. Le groupe $C r_{n}$ est, par définition, le groupe des automorphismes du corps des fonctions rationnelles à $n$ indéterminées. Si $n=1$. $C r_{1}$ coïncide avec le groupe des automorphismes de $\boldsymbol{P}_{1}$. Enriques a résolu ce problème pour $n=2$ (Enriques [5]). On croit généralement (par exemple Godeaux [8]) que Enriques et Fano l'ont fait pour $n=3$ (Enriques et Fano [6]). Après avoir collaboré avec Enriques, G. Fano a travaillé seul sur ce problème et il a laissé des résultats très intéressants dont la plupart, des démonstrations ne semble pas rigoureuse. Demazure [4] a étudie les: sous-groupes algébriques de rang maximum $n$ de $C r_{n}$. Il a démontré qu'il y a une correspondence bijective entre les sous-groupes algébriques de rang maximum $n$ de $\mathrm{Cr}_{n}$ et les systèmes d'Enriques. Puisque les derniers sont. de nature combinatoire, c'est un dictionaire géométrique-combinatoire. Mais il y a des sous-groupes algébriques maximaux dans $C r_{n}$ dont les rang sont $<n$, par exemple $\left(P S O_{5}\right.$, quadrique $\left.\subset \boldsymbol{P}_{4}\right)$ dans $C r_{3}$ (voir la remarque (3.15.2) et [14]).

Nous reprenons, dans cet article, le cas où $n=3$ et $G$ est primitif et retrouvons le résultat de Enriques et Fano [6] et Fano [7]. Une opération algébrique $(G, X)$ est dite primitive si $G$ ne laisse même localement aucun feullitage analytique invariant. Nous classifions birationnellement les

Received December 21, 1978. 
opérations algébriques primitives $(G, X)$ où $X$ est une variété algébrique rationnelle de dimension 3 . Toute telle opération est birationnellement isomorphe à une sous-opération algébrique de $\left(P G L_{4}, \boldsymbol{P}_{3}\right)$ et $\left(P S O_{5}\right.$, quadrique $\subset \boldsymbol{P}_{4}$ ) (théorème 3.4 et corollaire 3.14). En particulier, les opérations $\left(P G L_{4}, \boldsymbol{P}_{3}\right)$ et $\left(\mathrm{PSO}_{5}\right.$, quadrique $\left.\subset \boldsymbol{P}_{4}\right)$ determinent des classes de conjugaison maximales de sous-groupes algébriques connexes de $\mathrm{Cr}_{3}$. On peut démontrer directement la maximalité des opérations algébriques $\left(P G L_{4}, P_{3}\right)$ et $\left(P S O_{5}\right.$, quadrique $\subset \boldsymbol{P}_{5}$ ). D'ailleurs le théorème 3.4 est indipensable quand on étudie les sous-groupes algébriques imprimitifs de $\mathrm{Cr}_{3}$ (Umemura [14]).

Pour donner une démonstration au goût du jour, il faut distinguer les notions locales et globales ainsi que les notions analytiques et algébriques. Pour cette raison, aux premier et second paragraphes, nous rappelons les définitions connues pour rendre claires les termes utilisés. Notre classification birationnelle repose sur le théorème de Lie (Kapitel 7, § 36, Lie [9]) ainsi que la classification de Enriques et Fano [6]. D'abord, utilisant le théorème de Lie (la classification analytique locale), nous établissons la classification analytique globale. Puis nous démontrons que tout isomorphisme analytique global est algébrique.

\section{§1. Définitions analytiques}

DÉfINITION 1.1 (Bourbaki [3]). On appelle groupuscule analytique un système $(\mathrm{G}, \mathrm{e}, \theta, m)$ vérifiant les conditions suivantes:

(i ) $G$ est une variété analytique complexe;

(ii ) $e \in G$;

(iii) $\theta$ est une application analytique d'un voisinage de $e$ dans $G$;

(iv) $m$ est une application analytique d'une partie ouverte $\Omega$ de $G$ $\times G$ dans $G$;

( v ) pour tout $g \in G$, on a $(e, g) \in \Omega,(g, e) \in \Omega, m(e, g)=m(g, e)=g$;

( vi ) pour tout $g \in G$ tel que $\theta(g)$ soit dèfini, on a $(g, \theta(g)) \in \Omega,(\theta(g), g)$ $\in \Omega, m(g, \theta(g))=m(\theta(g), g)=e$;

(vii) $\mathrm{Si} g, h, k$ sont des éléments de $G$ tels que

$$
(g, h) \in \Omega, \quad(h, k) \in \Omega, \quad(m(g, h), k) \in \Omega, \quad(g, m(h, k)) \in \Omega,
$$

alors

$$
m(m(g, h), k)=m(g, m(h, k)) \text {. }
$$

L'élément $e$ s'appelle l'élément neutre de la groupuscule analytique. On écrit $g h$ au lieu de $m(g, h)$, et $g^{-1}$ au lieu de $\theta(g)$. 
DÉfinition 1.2. Soient $G_{1}, G_{2}$ deux groupuscules analytiques, d'éléments neutres $e_{1}, e_{2}$. Un morphisme de groupuscules analytiques $f$ de $G_{1}$ dans $G_{2}$ est une application analytique d'un sous-ensemble ouvert $U_{1}$ contenant $e_{1}$ dans $G_{2}$ vérifiant les conditions suivantes:

(i) $f\left(e_{1}\right)=e_{2}$;

(ii) Si $g, h$ dans $U_{1}$ sont tels que $g h$ soit défini et $g h$ soit un élément de $U_{1}$, alors $f(g) f(h)$ est défini et égal à $f(g h)$.

Le composé de deux morphismes de groupuscules analytiques est un morphisme de groupuscules analytiques. L'ensemble des groupuscules analytiques forme une catégorie. Elle est équivalente à la catégorie des algèbres de Lie de dimension fine.

Définition 1.3. On dit que deux groupuscules analytiques $G_{1}, G_{2}$ sont localement isomorphes s'il existe deux morphismes de groupuscules analytiques $f: G_{1} \rightarrow G_{2}$ et $f^{\prime}: G_{2} \rightarrow G_{1}$ tels que $f$ et $f^{\prime}$ sont des applications réciproques l'une de l'autre aux voisinages des éléments neutres.

Soient $(G, e, \theta, m)$ un groupuscule analytique, $X$ une variété complexe connexe.

DÉfinition 1.4. On appelle morceau de loi d'opération analytique à gauche de $G$ dans $X$ une application analytique $\psi$ d'une partie ouverte $\Omega$ de $G \times X$ contenant $\{e\} \times X$ dans $X$ vérifiant les propriétés suivantes:

(i) pour tout $x \in X$, on a $\psi(e, x)=x$;

(ii) il existe un voisinage $\Omega_{1}$ de $\{e\} \times\{e\} \times X$ dans $G \times G \times X$ tel que, pour $\left(g, g^{\prime}, x\right) \in \Omega_{1}$, les éléments $\left.m\left(g, g^{\prime}\right), \psi\left(g^{\prime}, x\right), \psi\left(m\left(g, g^{\prime}\right), x\right)\right)$, $\psi\left(g, \psi\left(g^{\prime}, x\right)\right)$ soient définis et $\psi\left(g, \psi\left(g^{\prime}, x\right)\right)=\psi\left(m\left(g, g^{\prime}\right), x\right)$.

On écrit $g x$ au lieu de $\psi(g, x)$.

Soit $U \neq \phi$ un sous-ensemble ouvert connexe de $X$. Alors $(G, X)$ induit sur $(G, U)$ une structure de morceau de loi d'opération analytique.

DÉfinITION 1.5. Un morceau de loi d'opération analytique à gauche est dit opération analytique à gauche si $G$ est un groupe analytique et $\psi$ est une application analytique de $G \times X$ dans $X$.

On écrit $g x$ au lieu de $\psi(g, x)$. Bien que la définition d'un morceau de loi d'opération dépende de $\psi$, nous utiliserons la notation $(G, X)$ sans préciser $\psi$. Puisque dans la suite on ne considère que des morceaux de lois d'opération analytique à gauche, on les appelle simplement morceaux de lois d'opèration analytique. 
DÉfinition 1.6. Soient $\left(G_{1}, X_{1}\right),\left(G_{2}, X_{2}\right)$ deux morceaux de lois d'opération analytique. Un morphisme de morceaux de lois d'opération analytique (ou un morphisme analytique local) $(\varphi, f)$ de $\left(G_{1}, X_{1}\right)$ dans $\left(G_{2}, X_{2}\right)$ consiste en un morphisme de groupuscules analytiques $\varphi$ de $G_{1}$ dans $G_{2}$ et une application analytique $f$ d'un sous-ensemble ouvert non vide de $X_{1}$ dans $X_{2}$ vérifiant la condition suivante: si $g \in G, x \in X_{1}$ tels que $g x, \varphi(g), f(x)$, $\varphi(g) t(x)$ et $f(g x)$ soient définis, alors $f(g x)=\varphi(g) f(x)$.

On dit que $\left(G_{1}, X_{1}\right)$ est isomorphe à $\left(G_{2}, X_{2}\right)$ en tant que morceaux de lois d'opération analytique s'il existe un morphisme local $(f, \varphi)$ de $\left(G_{1}, X_{1}\right)$ dans $\left(G_{2}, X_{2}\right)$ tel que $\varphi$ soit un isomorphisme local et $f$ soit un isomorphisme d'un ouvert de $X_{1}$ sur un ouvert de $X_{2}$.

Soient $\left(G_{1}, X_{1}\right),\left(G_{2}, X_{2}\right)$ deux opérations analytiques. Un morphisme d'opérations analytiques (ou morphisme analytique global) $(\varphi, f)$ est un morphisme analytique local $(\varphi, f)$ tel que $\varphi$ soit morphisme de groupe analytique, $f$ soit une application holomorphe et $f(g x)=\varphi(g) f(x)$ pour tout $g \in G_{1}$, tout $x \in X_{1}$.

Puisque l'on ne peut pas toujours définir le composé de deux morphismes analytiques locaux, l'ensemble des morceaux de lois d'opération analytique ne forme pas de catégorie. Pour la même raison, la notion d'isomorphisme local ne définit pas de relation d'équivalence.

Soit $(G, X)$ un morceau de loi d'opération analytique. Alors il induit un morphisme de l'algèbre de Lie $\mathfrak{g}$ de $G$ dans l'algèbre de Lie des champs de vecteurs holomorphes sur $X$ et inversement un morphisme de $g$ dans l'algèbre de Lie des champs de vecteurs holomorphes sur $X$ défini un morceau de loi d'opération analytique (voir Bourbaki [3]).

Définition 1.7. Un morceau de loi d'opération analytique $(G, X)$ est dit effectif si le morphisme de $g$ dans l'algébre de Lie des champs de vecteurs holomorphes sur $X$ est injectif.

Proposition 1.8. Soit $(G, X)$ un morceau de loi d'opération analytique. Il existe un morceau de loi d'opération analytique effectif $\left(G^{\prime}, X\right)$ et un morphisme de morceaux de loi d'opération analytique de $(G, X)$ dans $\left(G^{\prime}, X\right)$.

C'est une conséquence du théorème 6 III, $\S 4$ Bourbaki [3].

Soit $(G, X)$ une opération analytique. Nous aurons besoin de la condition suivante:

Condition 1.9. L'homomorphisme de $G$ dans Aut $X$ de groupes induit par opération est injectif (cf. définition 1.7). 
Proposition 1.10. Soit $(G, X)$ une opération analytique. Il existe une opération analytique $\left(G^{\prime}, X\right)$ satisfaisant à la condition (1.9) et un morphisme d'opérations analytiques de $(G, X)$ dans $\left(G^{\prime}, X\right)$.

Il suffit de considérer $(G / N, X)$ où $N$ est le noyau de $G \rightarrow$ Aut $X$ (cf. remarque (2.22).

Définition 1.11. Soit $(G, X)$ un morceau de loi d'opération analytique. On dit que $(G, X)$ est imprimitif s'il existe un morceau de loi d'opération analytique $(H, Y)$ et un morphisme de morceaux lois d'opération analytique $(\varphi, f)$ de $(G, X)$ dans $(H, Y)$ satisfaisant aux conditions suivantes: (1) il existe un point $x \in X$ tel que l'application $f$ soit definie en $x$ et la matrice jacobienne de $f$ ne s'annule pas en $x$. (2) $\operatorname{dim} X>\operatorname{dim} Y>0$. On dit que $(G, X)$ est primitif lorsque $(G, X)$ n'est pas imprimitif.

Par calcul, S. Lie [9] a classifié tous les morceaux de lois d'opération analytique effectif $(G, X)$ à relation d'équivalence engendrée par isomorphisme local près, si la dimension de $X \leq 2$. C'est un des principaux travaux de Lie. Lorsque la dimension de $X$ est égale à 3 , il a donné la liste exhaustive des morceaux de lois d'opération primitifs. De plus, il a étudié certains morceaux de lois d'opération imprimitifs de dimension 3.

THÉORÈme 1.12 (Lie [9]). Soit $(G, X)$ un morceau de loi d'opération analytique effectif. Si $(G, X)$ est primitif et la dimension de $X$ est 3 , alors $(G, X)$ est localement isomorphe à un des morceaux de lois d'opération analytique suivants:

(1) $\left(P G L_{4}, P_{3}\right)$;

(2) $\left(G T A_{3}, A_{3}\right)$;

(3) $\left(S G T A_{3}, A_{3}\right)$;

(4) $\left(P S p_{4}, P_{3}\right)$;

(5) $\left(P O_{4}, P_{3}\right)$;

(6) $\left(G T E_{3}, A_{3}\right)$;

(7) $\left(G S_{3}, A_{3}\right)$;

(8) $\left(P S O_{5},\left\{x_{0}^{2}+x_{1}^{2}+x_{2}^{2}+x_{3}^{2}+x_{4}^{2}=0\right\} \subset \boldsymbol{P}_{4}\right)$.

On trouve ci-dessous la définition des groupes: $G T A_{3}$ est le groupe des transformations affines. Autrement dit, c'est le sous-groupe de $P G L_{4}$ formé des matrices $\left(\begin{array}{ccc}1 & 0 & 0 \\ x & A\end{array}\right)$ où $x \in C^{3}, A \in G L_{3}$. GTA $A_{3}$ est un sous-groupe algébrique de $P G L_{4}$. Ainsi $G T A_{3}$ opère sur $P_{3}$ laissant invariant le plan 
infini et donc sur $\boldsymbol{P}_{3}-$ le plan infini $=\boldsymbol{A}_{3}$. Signalons que $\boldsymbol{A}_{3}$ est l'orbite ouverte de $P_{3}$ et $A_{3}$ est un espace homogène. $S G T A_{3}$ est le sous-groupe de $G T A_{3}$ formé des matrices $\left(\begin{array}{ccc}1 & 0 & 0 \\ x & A\end{array}\right) \in G T A_{3}$ telles que $A \in S L_{3} . \quad S O_{n}$ est le sous-groupe de $G L_{n}$ formé des matrices laissant invariant la forme quadratique $x_{1}^{2}+x_{2}^{2}+\cdots+x_{n}^{2}$. L'opération $\left(P S p_{4}, \boldsymbol{P}_{3}\right) \quad\left(\operatorname{resp} .\left(\mathrm{PSO}_{4}, \boldsymbol{P}_{3}\right)\right)$ est l'opération projectifiée de la représentation naturelle de degré $4 \mathrm{du}$ groupe symplectique (resp. orthogonal). $G T E_{3}$ est le groupe des transformations euclidiennes. C'est le sous-groupe de $S G T A_{3}$ formé des matrices

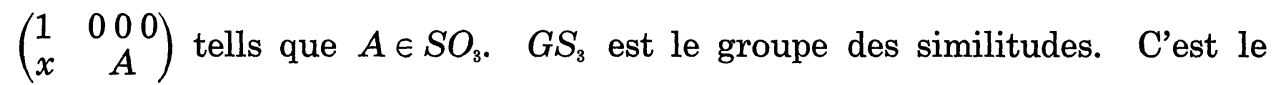

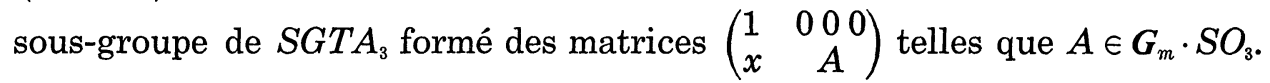
L'opération projectifiée $\left(\mathrm{PSO}_{5}, \boldsymbol{P}_{4}\right)$ de $\mathrm{SO}_{5}$ laisse invariante la quadrique $\left\{x_{0}^{2}+x_{1}^{2}+x_{2}^{2}+x_{3}^{2}+x_{4}^{2}+x_{5}^{2}=0\right\} \subset \boldsymbol{P}_{4}$. Ainsi le groupe $P S O_{5}$ opère sur la quadrique de dimension 3. Cette opération se note $\left(P_{S O_{5}},\left\{x_{0}^{2}+x_{1}^{2}+x_{2}^{2}+\right.\right.$ $\left.\left.x_{3}^{2}+x_{4}^{2}=0\right\} \subset \boldsymbol{P}_{4}\right)$ où $\left(P S O_{5}\right.$, quadrique $\left.\subset \boldsymbol{P}_{4}\right)$.

\section{§2. Définitions Algebriques}

Remplaçant des applications analytiques par applications régulières, on obtient la définition de groupuscule algébrique. Toute variété algébrique est définie sur un corps algébriquement clos $k$. Tout groupe algébrique est supposé connexe. D'ailleurs on considère des sous-groupes algébriques qui ne soient pas toujours connexes.

DÉfinition 2.1. On appelle groupuscule algébrique un système $(G, e$, $\theta, m)$ vérifiant les conditions suivantes:

(i) $G$ est une variété algébrique irreductible;

(ii) $e$ est un point fermé de $G$;

(iii) $\theta$ est une application rationnelle régulière à un voisinage de $e$;

(iv) $m$ est une application rationnelle de $G \times G$ dans $G$ régulière à une partie ouverte $\Omega$ de $G \times G$;

Les mêmes conditions (v), (vi) et (vii) que celles de la définition 1.1.

Comme au cas analytique, on écrit $g h$ au lieu de $m(g, h)$, et $g^{-1}$ au lieu de $\theta(g)$.

DÉfinition 2.2. Soient $G_{1}, G_{2}$ deux groupuscules algébriques d'éléments neutres $e_{1}, e_{2}$. Un morphisme de groupuscules algébriques $f$ de $G_{1}$ dans $G_{2}$ est une application rationnelle de $G_{1}$ dans $G_{2}$ vérifiant les conditions suivantes: 
(i ) $f$ est régulière à $e_{1}$ et $f\left(e_{1}\right)=e_{2}$;

(ii) Si $g, h$ dans $G_{1}$ sont tels que $g h$ soit défini et $f$ soit défini à $g h$, alors $f(g) f(h)$ est défini et égal à $f(g h)$.

Le composé de deux morphismes de groupuscules algébriques est un morphisme de groupuscules algébriques. L'ensemble des groupuscules algébriques forme une catégorie.

DÉFInITION 2.3. On dit que deux groupuscules algébriques sont birationnellement isomorphes s'il existe un morphisme de groupuscules algébriques qui est birationnel.

ThÉORغ̀me 2.4 (Weil [13]). Soit $G$ un groupuscule algébrique. Alors il existe un groupe algébrique $G^{\prime}$ birationnellement isomorphe à $G$.

La version analytique du théorème de Weil est le troisième théorème de Lie dont nous n'aurons pas besoin.

D'autre part, on a le théorème d'unicité.

ThÉORÈme 2.5 (Rosenlicht [11]). Soient $G_{1}, G_{2}$ deux groupes algébriques. Si $G_{1}$ est isomorphe à $G_{2}$ en tant que groupuscules algébriques, $G_{1}$ est isomorphe $\grave{a} G_{2}$ en tant que groupes algébriques.

DÉFInition 2.6. Soient $(G, e, \theta, m)$ un groupuscule algébrique, $X$ une variété algébrique irréductible. On appelle pseudo-opération algébrique (à gauche) de $G$ sur $X$ une application rationnelle $\psi$ de $G \times X$ dans $X$ vérifiant les conditions suivantes:

(i) pour un point général $x \in X_{1}$, l'application $\psi$ soit régulière à $(e, x)$ et $\psi(e, x)=x$.

(ii) pour deux points généraux $g, g^{\prime} \in G$ et un point général $x \in X$, on a $\psi\left(g,\left(g^{\prime}, x\right)\right)=\psi\left(m\left(g, g^{\prime}\right), x\right)$.

Lorsque $G$ est un groupe algébrique, on dit que $(G, X)$ ou parfois $X$ est une pseudo-opération algébrique du groupe algébrique $G$ sans preciser $\psi$. On écrit $g x$ au lieu de $\psi(g, x)$.

Une pseudo-pération algébrique $(G, X)$ est dite morceau de loi d'opération algébrique si $\psi$ est régulière sur une partie ouverte $\Omega$ de $G \times X$ contenant $\{e\} \times X$.

Soient $(G, X)$ une pseudo-opération algébrique (resp. un morceau de loi d'opération algébrique) et $U \neq \phi$ un sous-ensemble ouvert de $X$. Alors $(G, U)$ porte la structure de pseudo-opération algébrique (resp. morceau de loi d'opération algébrique) induite par $(G, X)$. 
DÉFINITION 2.7. Un morceau de loi d'opération algébrique est dit opération algébrique si $G$ est un groupe algébrique et $\psi$ est un morphisme de variétés algébriques de $G \times X$ dans $X$.

DÉfinition 2.8. Soient $\left(G_{1}, X_{1}\right),\left(G_{2}, X_{2}\right)$ deux pseudo-opérations algébriques (resp. morceaux de lois d'opération algébrique). Un morphisme de pseudo-opérations algébriques (resp. morceaux de lois d'opération algébrique) $(\varphi, f)$ de $\left(G_{1}, X_{1}\right)$ dans $\left(G_{2}, X_{2}\right)$ consiste en un morphisme de groupuscules algébriques $\varphi$ de $G_{1}$ dans $G_{2}$ et une application rationnelle $f$ de $X_{1}$ dans $X_{2}$ vérifiant la condition suivante; si $g \in G_{1}, x \in X_{1}$ tels que $g x$, $\varphi(g), f(x), \varphi(g) f(x)$ et $f(g x)$ soient definis, alors $f(g x)=\varphi(g) f(x)$.

On dit que $\left(G_{1}, X_{1}\right)$ est isomorphe en tant que pseudo-opération algébrique (resp. morceau de loi d'opération algébrique) ou birationnellement isomorphe à $\left(G_{2}, X_{2}\right)$ s'il existe un morphisme de pseudo-opérations algébriques (resp. morceaux de lois d'opération algébrique $(\varphi, f)$ tel que $\varphi$ et $f$ soient birationnels.

Soient $\left(G_{1}, X_{1}\right),\left(G_{2}, X_{2}\right)$ deux opérations algébriques. Un morphisme d'opérations algébriques $(\varphi, f)$ de $\left(G_{1}, X_{1}\right)$ dans $\left(G_{2}, X_{2}\right)$ est un morphisme de morceaux de lois d'opération algébrique de $\left(G_{1}, X_{1}\right)$ dans $\left(G_{2}, X_{2}\right)$ tel que $\varphi$ soit un morphisme de groupes algébriques, $f$ une application régulière et $f(g x)=\varphi(g) f(x)$ pour tout $g \in G_{1}$, tout $x \in X_{1}$.

Le théorème suivant réduit l'étude des pseudo-opérations algébriques et morceaux de lois d'opération algébrique en celle des opérations algébriques.

ThÉonغ̀me 2.9 (Weil [13]). Soit $(G, X)$ une pseudo-opération algébrique. Alors il existe une opération algébrique $\left(G^{\prime}, X^{\prime}\right)$ telle que $(G, X)$ soit birationnellement isomorphe à $\left(G^{\prime}, X^{\prime}\right)$.

Pour l'unicité de $\left(G^{\prime}, X^{\prime}\right)$, on a le résultat suivant:

ThÉoRغ̀me 2.10 (Rosenlicht [11]). Soient $\left(G_{1}, X_{1}\right),\left(G_{2}, X_{2}\right)$ deux opérations algébriques. Si $\left(G_{1}, X_{1}\right)$ est isomorphe à $\left(G_{2}, X_{2}\right)$ en tant que morceau de loi d'opération algébrique, alors, pour chaque $i$, il existe une partie ouverte $U_{i} \neq \phi$ de $X_{i}$ telle que $U_{i}$ soit invariante par $G_{i}$ et $\left(G_{1}, U_{1}\right)$ soit isomorphe $\grave{a}\left(G_{2}, U_{2}\right)$ en tant qu'opérations algébriques.

Soit $X$ une variété algébrique. On designe par Aut birat $X$ le groupe des automorphismes birationnels de $X$. C'est le groupe des $k$-automorphismes du corps des fonctions rationnelles $k(X)$. En générale, Aut birat $X$ 
n'est pas un groupe algébrique. Mais on peut parler d'un sous-groupe algébrique de Aut birat $X$. Pour définir correctement un sous-groupe algébrique de Aut birat $X$, il faut interpréter Aut birat $X$ comme un foncteur de la catégorie des variétés algébriques sur $k$ dans la catégorie des groupes (Demazure [4]).

DÉfinition 2.11. Soit $G$ un groupe algébrique. Donc $G$ est un foncteur de la catégorie des variétés algébriques sur $k$ dans la catégorie des groupes. Un homomorphisme algébrique $\varphi$ de $G$ dans Aut birat $X$ est un morphisme de foncteurs de $G$ dans Aut birat $X$. On dit que $G$ est un sous-groupe algébrique de Aut birat $X$ si l'homomorphisme $\varphi$ est injectif, i.e. $G$ est un sous-foncteur de Aut birat $X$.

Proposition 2.12 (Demazure [4]). Il existe une correspondence bijective entre les homomorphismes algébriques de $G$ dans Aut birat $X$ et les pseudoopérations algébriques de $G$ sur $X$.

Proposition 2.13 (Demazure [4]). Soit $\varphi: G \rightarrow$ Aut birat $X$ un homomorphisme algébrique. Le noyau $N$ de $\varphi$ est un sous-groupes algébrique de $G$.

CoRollaIRe 2.14. Soit $(G, X)$ une pseudo-opération algébrique d'un groupe algébrique $G$. Il existe une opération algébrique $\left(G^{\prime}, X\right)$ d'un groupe algébrique $G^{\prime}$ définissant un sous-groupe algébrique de Aut briat $X$ et un morphisme d'opérations algébriques de $(G, X)$ dans $\left(G^{\prime}, X\right)$.

Soit $N$ le noyau de l'homomorphisme $G \rightarrow$ Aut birat $X$. Il suffit de considerer l'homomorphisme algébrique $G^{\prime}=G / N \rightarrow$ Aut birat $X$.

Soit $(G, X)$ une pseudo-opération algébrique d'un groupe algébrique $G$.

Condition 2.15. L'homomorphisme de groupes abstraits de $G(C)$ dans Aut birat $X(C)$ est injectif.

Si $(G, X)$ est une opération algébrique, cette condition est equivalente à

Condition 2.16. L'homomorphisme de groupes abstraits de $G(C)$ dans Aut $X(C)$ est injectif.

CoRollaIRe 2.17. On suppose $k=C$. Un homomorphisme algébrique $G \rightarrow$ Aut briat $X$ defini par une opération algébrique $(G, X)$ est injectif si et seulement si $(G, X)$ satisfait à la condition 2.16 . 
En effect, tout groupe algébrique en caractéritique 0 est réduit et le noyau $N$ est trivial si et seulement si $N(C)$ est trivial.

On suppose desormais $k=C$. D'après le corollaire 2.17 il suffit de considerer les pseudo-opérations algébriques des groupes algébriques satisfaisant à la condition ensembliste 2.15 au lieu de sous-groupes algébriques de Aut birat $X$.

Proposition 2.18. Il existe une correspondence bijective entre les classes de conjugaison des sous-groupes algébriques de Aut birat $X$ et les classes d'isomorphisme birationnel des opérations algébriques $\left(G, X^{\prime}\right)$ satisfaisant à la condition 2.16 telles que $X^{\prime}$ soit birationnellement isomorphe $\grave{a} X$.

Soit $\left(G, X^{\prime}\right)$ une opération algébrique satisfaisant à la condition 2.16 telle qu'il existe un isomorphisme birationnel $f: X^{\prime} \rightarrow X$. L'isomorphisme birationnel $f$ define une pseudo-opération algébrique de $G$ sur $X$ donc un sous-groupe algébriqué de Aut birat $X$. Si l'on remplace $f$ par un autre isomorphisme birationnel $f^{\prime}$, le sous-groupe algébrique de Aut birat $X$ défini par $f^{\prime}$ est conjugué à celui défini par $f$. D'après le théorème 2.9 et la proposition 2.12 tout sous-groupe algébrique est ainsi défini. Deux sousgroupes algèbriques $G_{1}, G_{2}$ de Aut birat $X$ sont conjugués si et seulement si les pseudo-opérations algébriques des groupes algébriques $\left(G_{1}, X\right),\left(G_{2}, X\right)$ sont birationnellement isomorphes.

Soit $X$ une variété algébrique (sur $C$ ). On désigne par $X^{a n}$ l'espace analytique associé a $X$. Soit $f$ un morphisme de variétés algébriques. Le morphisme analytique associé est noté par $f^{a n}$. Soit $(G, X)$ un morceau de loi d'opération algébrique (resp. une opération algébrique). Alors, le morceau de loi d'opération analytique associé (resp. l'opération analytique associeé) est désigné par $\left(G^{a n}, X^{a n}\right)$.

Proposition 2.19. Soient $G$ un groupe algébrique et $(G, X)$ un morceau de loi d'opération algébrique. Si $\left(G^{a n}, X^{a n}\right)$ est primitif, alors, pour tout morceau de loi d'opération algébrique $(G, Y)$ birationnellement isomorphe à $(G, X),\left(G^{a n}, Y^{a n}\right)$ est prmitif.

En effect, soit $(\varphi, f):(G, X) \rightarrow(G, Y)$ un isomorphisme birationnel. Le morphisme $f^{a n}$ est un isomorphisme d'une partie ouverte de $X$ dans une partie ouverte de $Y$. La proposition resulte de la définition 1.11 et du fait que toute partie ouverte non vide pour la topologie de Zariski est dense.

DÉFInITION 2.20. Soit $(G, X)$ une opération algébrique definissant une 
classe de conjugaison $C$ de Aut birat $X$. Lorsque l'opération analytique $\left(G^{a n}, X^{a n}\right)$ est primitive, on dit que la classe de conjugaison $C$ est primitive. Tout sous-groupes algébrique dans $C$ est dit primitif.

Si l'opération analytique $\left(G^{a n}, X^{a n}\right)$ est primitive, alors d'après la proposition 2.19, pour toute opération algébrique $(G, Y)$ definnissant la classe de conjugaison $C$, l'opération analytique $\left(G^{a n}, Y^{a n}\right)$ est primitive.

Proposition 2.21. Soient $\left(G_{i}, X_{i}\right)(i=i, 2)$ deux opérations algébriques (sur $C$ ) satisfaisant aux condition suivantes:

(1) $\left(G_{i}, X_{i}\right)(i=1,2)$ satisfont à la condition 2.16 ;

(2) il existe un isomorphisme d'opérations analytiques $\left(\varphi^{\prime}, f^{\prime}\right)$ de $\left(G_{1}^{a n}, X_{1}^{a n}\right)$ dans $\left(G_{2}^{a n}, X_{2}^{a n}\right)$;

(3) le morphisme f'est algébrique; il existe un isomorphisme $f$ de variétés algébriques de $X_{1}$ dans $X_{2}$ tel que $f^{a n}=f^{\prime}$;

(4) il existe un complété equivariant $\left(G_{2}, \bar{X}_{2}\right)$ de $\left(G_{2}, X_{2}\right) ; \bar{X}_{2}$ est une variété projective lisse et il existe une immersion ouverte $i: X_{2} \rightarrow \bar{X}_{2}$ telle que $(\mathrm{Id}, i):\left(G_{2}, X_{2}\right) \rightarrow\left(G_{2}, \bar{X}_{2}\right)$ soit un morphisme d'opérations algébriques.

Alors, l'opération $\left(G_{1}, X_{1}\right)$ est isomorphe $\grave{a}\left(G_{2}, X_{2}\right)$ en tant qu'opération algébrique.

Posant $g_{1} x_{2}=f g_{1} f^{-1}\left(x_{2}\right)$ pour $g_{1} \in G_{1}, x_{2} \in X_{2}$, on obtient une opération algébrique. Il suffit, donc, de montrer que $\left(G_{1}, X_{2}\right)$ est isomorphe à $\left(G_{2}, X_{2}\right)$ en tant qu'opération algébrique. Puisque l'opération $\left(G_{1}^{a n}, X_{2}^{a n}\right)$ est isomorphe à $\left(G_{2}^{a n}, X_{2}^{a n}\right)$, la loi d'opération $\left(G_{1}, X_{2}\right)$ se prolonge à une opération analytique $\left(G_{1}^{a n}, \bar{X}_{2}^{a n}\right)$. On en conclut que le morphisme de variétés algébriques $\psi\left(g_{1}, x_{2}\right) \mapsto g_{1} x_{2}$ de $G_{1} \times X_{2}$ dans $X_{2}$ se prolonge à un morphisme de variétés analytiques $\bar{\psi}\left(g_{1}, x_{2}\right) \mapsto g_{1} x_{2}$ de $\left(G_{1} \times X_{2}\right)^{a n}$ dans $X_{2}^{a n}$. Le morphisme $\bar{\psi}$ est algebrique parce qu'il coïncide au morphisme algébrique $\psi$ sur l'ouvert pour la topologie de Zariski $G_{1} \times X_{2}$ de $G_{1} \times \bar{X}_{2}$. Puisque la variété $\bar{X}_{2}$ est projective, Aut $\bar{X}_{2}$ est un groupe algébrique. Donc, on a montré que $\left(G_{1}, X_{2}\right),\left(G_{2}, X_{2}\right)$ sont des sous-opérations de (Aut $\bar{X}_{2} . \bar{X}_{2}$ ). Les groupes algébriques $G_{1}, G_{2}$ sont des sous-groupes algébriques du groupe algébrique Aut $\bar{X}_{2}$ et les ensembles des points rationnels $G_{1}(C)$ et $G(C)$ se coincident. Puisque $G_{1}$ et $G_{2}$ sont reduits, $G_{1}$ coincide avec $G_{2}$. Donc, l'opération $\left(G_{1}, X_{2}\right)$ est isomorphe à $\left(G_{2}, X_{2}\right)$ en tant qu'opération algébrique.

Remarque 2.22. On ignore si la condition 4 de la proposition 2.21 est nécessaire. La proposition 2.21 se resute plus facilement de l'enoncé 
suivant sans supposer la condition 4:

2.23. Soient $G_{1}, G_{2}$ deux sous-groupes algébriques de Aut birat $X$. Alors, 1 intersection $G_{1} \cap G_{2}$ est un sous-groupe algébrique de Aut birat $X$.

Ou plus généralement,

2.24. Soient $G_{i} i=1,2$ deux groupes algébriques et $f_{i}: G_{i} \rightarrow$ Aut birat $X$ $i=1,2$ deux morphismes de foncteurs. Alors le produit fibré $G_{1} \times$ Aut briat $x G_{2}$ est représentable.

On croit que 2.24 est vrai mais on n'a pas reussi à montrer.

\section{§3. Classification Algébrique}

Soient $\tilde{G}, G$ deux groupes analytiques, $\varphi: \tilde{G} \rightarrow G$ un morphisme de groupes analytiques, $H$ un sous-groupe analytique fermé de $G$. Soient $\tilde{g}$ l'algèbre de Lie de $\tilde{G}, \mathfrak{g}$ l'algèbre de Lie de $G, \mathfrak{h}$ l'algèbre de Lie de $H$. h est une sous-algèbre de $g$ et $\varphi$ induit un morphisme $\varphi_{*}: \tilde{g} \rightarrow \mathfrak{g}$ d'algèbres de Lie.

LEMme 3.1. Si tout sous-groupe analytique fermé $\tilde{H}$ de $\tilde{G}$ tel que l'algèbre de Lie de $\tilde{H}$ soit $\varphi_{*}^{-1}(\mathfrak{G})$ est connexe, alors (i) tout $\tilde{H}$ coïncide avec $\varphi^{-1} H$; (ii) il existe un morphisme $(\varphi, f):(\tilde{G}, \tilde{G} / \tilde{H}) \rightarrow(G, G / H)$ d'opérations analytiques; (iii) $\tilde{G} / \tilde{H}$ est isomorphe à $G / H$ en tant que variété analytique.

Le premier énoncé est trivial. $G$ opère sur $G / H$ par morphisme $\varphi$. Soit $K$ le stabilisateur de $\tilde{G}$ à $H \in G / H$. On a un morphisme d'opérations analytiques de $(\tilde{G}, \tilde{G} / K)$ dans $(G, G / H)$ et $\tilde{G} / K$ est isomorphe à $G / H$ en tant que variété analytique. Comme l'algèbre de Lie de $K$ est $\varphi_{*}^{-1}(\mathfrak{h})$, il résulte de l'hypothèse que $K=\tilde{H}$.

Soient $G_{1}, G_{2}$ deux groupes analytiques. Soit $H_{i}$ un sous-groupe analytique fermé de $G_{i}$ pour $i=1,2$. S'il existe un isomorphisme $(\varphi, f):\left(G_{1}, G_{1} / H_{1}\right)$ $\rightarrow\left(G_{2}, G_{2} / H_{2}\right)$ de morceaux de lois d'opération analytique, on peut supposer que $f$ est défini à $H_{1} \in G / H_{1}$ et $f\left(H_{1}\right)=H_{2}$.

LEMmE 3.2. Soient $G_{i}(i=1,2)$ un groupe analytique, $H_{i}$ un sous-groupe analytique fermé de $G_{i},(\varphi, f):\left(G_{1}, G_{1} / H_{1}\right) \rightarrow\left(G_{2}, G_{2} / H_{2}\right)$ un isomorphisme de morceaux de lois d'opération analytique tels que $f$ soit défini à $H_{1}$ et $f\left(H_{1}\right)$ $=H_{2}$. On suppose qu'il existe un groupe analytique $\tilde{G}$, des morphismes de groupes analytiques: $\varphi_{i}: \tilde{G} \rightarrow G_{i}$ tels que $\varphi_{2 *}=\varphi_{*} \circ \varphi_{1 *}$ et $\varphi_{1}$ satisfasse à la condition du lemme 3.1. Si, pour chaque $i,\left(G_{i}, G_{i} / H_{i}\right)$ satisfait à la condition $1.9,\left(G_{1}, G_{1} / H_{1}\right)$ est isomorphe $\grave{a}\left(G_{2}, G_{2} / H_{2}\right)$ en tant qu'opération analytique. 
En vertu de la démonstration du lemme 3.1, il existe un morphisme d'opérations analytiques $\left(\varphi, f_{i}\right):\left(\tilde{G}, \tilde{G} / \tilde{H}_{i}\right) \rightarrow\left(G_{i}, G_{i} / H_{i}\right)$ pour chaque $i$ où $\tilde{H}_{i}=\varphi_{i}^{-1}\left(H_{i}\right)$.

Il résulte de l'hypothèse $\varphi_{2 *}=\varphi_{*} \circ \varphi_{1 *}$ que l'algèbre de Lie de $\tilde{H}_{1}$ coïncide avec l'algèbre de Lie de $\tilde{H}_{2}$. D'après hypothèse sur $\varphi_{i}$ et $H_{i}$, on a $\tilde{H}_{1}=\tilde{H}_{2}=\tilde{H}_{1}^{0}=\tilde{H}_{2}^{0}$. On posera $\tilde{H}_{1}^{0}=\tilde{H}_{2}^{0}=\tilde{H}$. Donc $\tilde{G} / \tilde{H}_{i}=\tilde{G} / \tilde{H}$. D'ailleurs, si l'on pose pour chaque $i \tilde{K}_{i}=$ noyau de $\varphi_{i},\left(\tilde{G} / \tilde{K}_{i},\left(\tilde{G} / \tilde{K}_{i}\right) /\left(\tilde{H} / \tilde{K}_{i}\right)\right)$ est isomorphe à $\left(G_{i}, G_{i} / H_{i}\right)$ en tant qu'opération analytique. Puisque $\left(G_{i}, G_{i} / H_{i}\right)$ satisfait à la condition 1.9 et $f_{i}$ est bijectif, $\tilde{K}_{i}=$ noyau de morphisme de $\tilde{G}$ dans Aut $\tilde{G} / \tilde{H}_{i}$ induit par opération de $\tilde{G}$. Puisque nous avons montré que $\tilde{H}_{1}=\tilde{H}_{2}$, donc $\tilde{K}_{1}=\tilde{K}_{2}$ que nous noterons $\tilde{K}$. Par conséquent $\left(G_{i}, G_{i} / H_{i}\right)$ est isomorphe à $\left.(\tilde{G} / \tilde{K},(\tilde{G} / \tilde{K}) /(\tilde{H}) \tilde{K})\right)$ en tant qu'opération analytique.

Lemme 3.3. Soient $G_{1}, G_{2}$ deux groupes analytiques. Soit $H_{i}$ un sousgroupe analytique fermé de $G_{i}$ pour $1 \leq i \leq 2$. On suppose que $\left(G_{1}, G_{1} / H_{1}\right)$ est isomorphe à $\left(G_{2}, G_{2} / H_{2}\right)$ en tant que morceau de loi d'opération analytique. Soient $\mathrm{g}, \mathfrak{h}$ les algèbres de Lie de $G_{i}, H_{i}$. Soit $\tilde{G}$ le groupe analytique simplement connexe d'algèbres de Lie g. Si tout sous-groupe analytique fermé $\tilde{H}$ de $\tilde{G}$ d'algèbre de Lie $\mathfrak{h}$ est connexe, et si, pour $i=1,2$, $\left(G_{i}, G_{i} / H_{i}\right)$ satisfait à la condition 1.9 , alors $\left(G_{1}, G_{1} / H_{1}\right)$ est isomorphe à $\left(G_{2}, G_{2} / H_{2}\right)$ en tant qu'opérations analytiques.

En effet, c'est un cas particulier du lemme 3.2.

Soit $(G, X)$ un morceau de loi d'opération algébrique. D'après le théorème 2.9, il existe une opération algébrique $\left(G^{\prime}, X^{\prime}\right)$ et un isomorphisme birationnel $(\varphi, f):(G, X) \rightarrow\left(G^{\prime}, X^{\prime}\right)$ de morceaux de lois d'opération algébrique. Si pour tout point général $x$ de $X$ et $g \in G$, on pose $\psi(x)=f^{-1} \circ L_{g}$ 。 $f(x)^{(1)}, \psi$ est un homomorphisme de groupes de $G^{\prime}$ dans le groupe Aut birat $(X)$ des automorphismes birationnels de $X$. Le groupe, l'image de $G^{\prime}$ dans Aut birat $(X)$, dépend de $f$ mais il est bien déterminé à conjugaison près.

Le groupe des automorphismes birationnels de l'espace projectif de dimension $n$ s'appelle groupe de Cremona et se note $C r_{n}$. Le problème suivant est classique; classifier à conjugaison près les sous-groupes algébriques connexes maximaux de $\mathrm{Cr}_{n}$.

D'après la proposition 2.18 , si $n=3$, le problème est équivalent à la

(1) $L_{g}$ est l'automorphisme de $X$ défini par opération de $g$. 
classification birationnelle des opérations algébriques satisfaisant à la condition 2.16 sur les variétés algébriques rationnelles et de dimension 3 .

THÉORÈme 3.4. Soient $G$ un groupe algébrique, $X$ une variété rationnelle de dimension 3 sur $C,(G, X)$ une opération algébrique satisfaisant à la condition 2.16. Si l'opération analytique associée $\left(G^{a n}, X^{a n}\right)$ est primitive, alors $(G, X)$ est birationnellement isomorphe à un des morceaux de lois d'opération algébrique figurant dans la liste ci-dessous.

(1) $\left(P G L_{4}, \boldsymbol{P}_{3}\right)$,

(2) $\left(G T A_{3}, A_{3}\right)$,

(3) $\left(S G T A_{3} A_{3}\right)$,

(4) $\left(\mathrm{PSp}_{4}, \boldsymbol{P}_{3}\right)$,

(5) $\left(\mathrm{PSO}_{4}, \boldsymbol{P}_{3}\right)$,

(6) $\left(G T E_{3}, A_{3}\right)$,

(7) $\left(G S_{3}, A_{3}\right)$,

(8) $\left(P S O_{5},\left\{x_{0}^{2}+x_{1}^{2}+x_{2}^{2}+x_{3}^{2}+x_{4}^{2}=0\right\} \subset \boldsymbol{P}_{4}\right)$,

(9) $\left(\mathrm{SO}_{4}, \mathrm{SO}_{4} / \mathrm{K}\right)$, où $\mathrm{K}$ est le sous-groupe algèbrique formé des matrices

$$
\left(\begin{array}{llll}
1 & 0 & 0 & 0 \\
0 & & & \\
0 & & A \\
0 & &
\end{array}\right)
$$

de $\mathrm{SO}_{4}$.

Puisque $\left(G^{a n}, X^{a n}\right)$ est primitif, $\left(G^{a n}, X^{a n}\right)$ est génériquement transitif. $(G$, l'orbite ouverte) est birationnellement isomorphe à $(G, X)$. On peut supposer que $(G, X)$ est un espace homogène $(G, G / H)$. D'autre part, $\left(G^{a n}, X^{a n}\right)$ est isomorphe en tant que morceau de loi d'opération analytique à un des $(1),(2), \cdots,(8)$ du théorème 1.12 .

Cas 3.5 où $\left(G^{a n}, X^{a n}\right)=\left(G^{a n},(G / H)^{a n}\right)$ est isomorphe, en tant que morceau de loi d'opération analytique, à (1), (4) ou (8) du théorème 1.12. Alors $G$ est semi-simple et $H$ est un sous-groupe parabolique. Puisque tout sous-groupe parabolique d'un groupe de Lie semi-simple est connexe, il résulte du lemme 3.3 que $\left(G^{a n},(G / H)^{a n}\right)$ est isomorphe à l'une opérations analytiques (1), (4). (8) du théorème 1.12. D'autre part, on sait que tout homomorphisme de groupes de Lie semi-simples est algébrique (voir 3.10). Il en résulte que $(G, G / H)$ est isomorphe en tant qu'opération algébrique à l'une des opérations algébriques (1), (4). (8) du théorème 1.12. 
Cas $3.6^{1)}$ où $\left(G^{a n}, X^{a n}\right)=\left(G^{a n},(G / H)^{a n}\right)$ est isomorphe, en tant que morceau de loi d'opération analytique à un des (2). (3), (6), (7) du théorème 1.12. Puisque la démonstration est identique, on peut supposer que $\left(G^{a n}, X^{a n}\right)=\left(G^{a n},(G / H)^{a n}\right)$ est isomorphe à (6) du théorème 1.12 en tant que morceau de loi d'opération analytique. Soient $G_{1}$ le groupe des transformations euclidiennes $G T E_{3}, \mathfrak{g}_{1}$ son algèbre de Lie, $H_{1}$ le stabilisateur de $G_{1}$ à ${ }^{t}(0,0,0) \in A_{3}$ (ou à $\left.{ }^{t}(1,0,0,0) \in P_{3}\right)$, h l'algèbre de Lie de $H_{1} . \quad\left(G_{1}, A_{3}\right)$ est isomorphe, en tant qu'opération algébrique à $\left(G_{1}, G_{1} / H_{1}\right)$. L'algèbre $g_{1}$ est une sous-algèbre de $s \ell_{4}$ des matrices $x \in s \ell_{4}$ telles que $x_{i j}=0$ pour $1 \leq j$ $\leq 4$ et $\left(x_{i j}\right)_{i, j \geq 2} \in S O_{3}$ et $\mathfrak{h}$ la sous-algèbre de $\mathfrak{g}_{1}$ des matrices $x \in \mathfrak{g}$, telles que $x_{i 1}=0$ pour $1 \leq i \leq 4$.

Afin de pouvoir appliquer le lemme 3.3, montrons d'abord quelques lemmes.

Lemme 3.7. Tout sous-groupe analytique fermé $L$ de $G_{1}$ tel que l'algèbre de Lie de $L$ soit $\mathfrak{h}$ est connexe.

En effet, par hypothèse

$$
L^{0}=\left\{x \in G_{1} \mid x_{11}=1, x_{1 \ell}=x_{\ell 1}=02 \leq \ell \leq 4,\left(x_{i j}\right)_{i, j \geq 2} \in S O_{3}\right\} .
$$

Supposons par absurde que $L$ n'est pas connexe. Alors il existe un élément

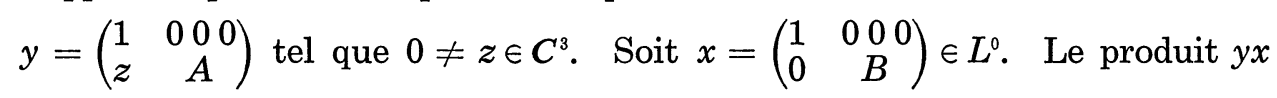

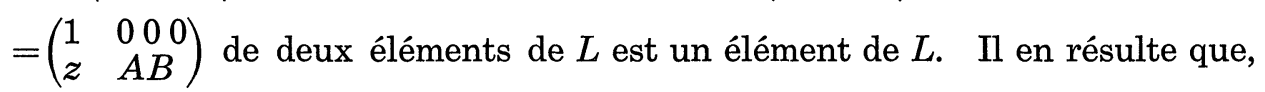

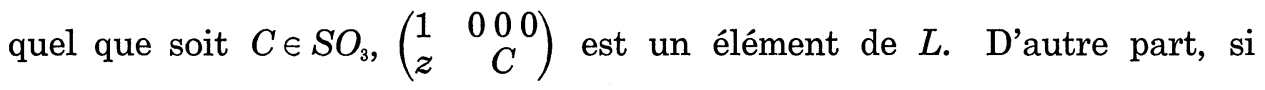
$D \in S_{3}$, le produit

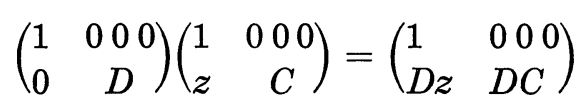

de deux éléments est un élément de $L$. Par conséquent, quels que soient $E, F \in S O_{3},\left(\begin{array}{ll}1 & 0 \\ E z & F\end{array}\right)$ est un élément de $L$. Donc, quels que soient $A, B, A^{\prime}$, $B^{\prime} \in S_{3}$, le produit

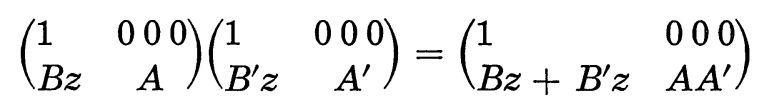

est un élément de $L$. Cela montre que le groupe analytique $L$ a un paramètre dans la première colonne, ce qui est impossible.

1) Une demonstration plus simple se trouve dans Umemula [15]. 
Le groupe analytique $G_{1}$ est homéomorphe à $C^{3} \times S_{3}$. Il en résulte que le groupe fondamental $\pi_{1}\left(G_{1}\right)$ est isomorphe à $Z / 2 Z$. Si $\tilde{G}$ désigne le revêtement universel de $G_{1}$, la projection $\varphi_{1}$ est un morphisme de groupes analytiques de degré 2.

Lemme 3.8. Tout sous-groupe analytique fermé $\tilde{H}$ de $\tilde{G}$ tel que l'algèbre de Lie de $\tilde{H}$ soit $\varphi_{1 *}^{-1}(\mathfrak{h})$ est connexe.

Le groupe algébrique $\varphi_{1}(\tilde{H})$ est connexe et égal à $H_{1}$ d'après le lemme 3.7. On a donc $\varphi_{1}^{-1}\left(H_{1}\right) \supset \tilde{H}$. Il existe un morphisme de variétés analytiques

$$
\tilde{G} / \tilde{H}^{0} \stackrel{\alpha}{\longrightarrow} \tilde{G} / \tilde{H} \stackrel{\beta}{\longrightarrow} \tilde{G} / \varphi_{1}^{-1}(H)=G / H_{1} .
$$

D'autre part $\left(G_{1} / H_{1}\right)^{a n}$ est homéomorphe à $C^{3}$ donc simplement connexe. Il en résulte que le degré de $\beta \circ \alpha$ est 1 . Autrement dit $\tilde{H}^{0}=\tilde{H}=\varphi_{1}^{-1}\left(H_{1}\right)$.

$$
\text { c.q.f.d. }
$$

On peut appliquer maintenant le lemme 3.3 et on en conclut qu'il existe un isomorphisme d'opération analytiques: $(\varphi, f):\left(G^{a_{n}},(G / H)^{a n}\right)=$ $\left(G^{a n}, X^{a n}\right) \rightarrow\left(G_{1}^{a n},\left(G_{1} / H_{1}\right)^{a n}\right)=\left(G T E_{3}, A_{3}\right)$. Il faut algébriser l'isomorphisme analytique $(\varphi, f)$. D'après la proposition 2.21, il suffit de montrer que $f$ est algébrique. Soit $R$ le radical de $G$. Puisque le radical de $G T E_{3}$ est commutatif, $R$ est commutatif. Donc $R$ est isomorphe au produit $\boldsymbol{G}_{m}^{r} \times \boldsymbol{G}_{a}^{s}$, $r, s \in N$ en tant que groupe algébrique. Le groupe analytique $R^{a n}$ est isomorphe au radical de $G T E_{3}$ qui est isomorphe à $G_{a}^{3}$. On en conclut $r=0$ $s=3$ et $R$ est isomorphe à $G_{a}^{3}$ en tant que groupe algébrique. La variété algébrique $X$ est un espace homogène de $R=G_{a}^{3}$. Si l'on restreint l'isomorphisme analytique $(\varphi, f)$ à $(R, X)$, on obtient $\left.(\varphi, f)\right|_{(R, X)}:\left(R^{a n}, X^{a n}\right)=$ $\left(\left(\boldsymbol{G}_{a}^{3}\right)^{a n},\left(\boldsymbol{G}_{a}^{3}\right)^{a n}\right) \rightarrow\left(\left(\boldsymbol{G}_{a}^{3}\right)^{a n}, \boldsymbol{A}_{3}^{a n}\right)$. On en conclut que $f$ est une transformation affine donc algébrique.

Il ne reste qu'à faire la vérification dans le cas 3.9 où $\left(G^{a n}, X^{a n}\right)$ est isomorphe à $\left(\mathrm{PSO}_{4}, \boldsymbol{P}_{3}\right)$ en tant que morceau de loi d'opération analytique.

Rappelons le fait suivant:

3.10. (a) Tout group analytique semi-simple est algébrique.

(b) Soient $A$ un groupe algébrique semi-simple, $B$ un groupe algébrique. Alors on a $\operatorname{Hom}_{a l g}(A, B)=\operatorname{Hom}_{a n}\left(A^{a n}, B^{a n}\right)$ où $\operatorname{Hom}_{a l g}(),\left(\operatorname{resp} . \operatorname{Hom}_{a n}(),\right)$ désigne l'ensemble des homomorphismes de groupes algébriques (resp. analytiques). 
Voir chap. VIII, Serre [14].

Si $x$ désigne le point ${ }^{t}(1,0,0,0) \in \boldsymbol{P}_{3}$, l'orbite de $x$ est de dimension 3 . Le stabilisateur $H$ de $x$ est formé des matrices

$$
\left(\begin{array}{cccc}
(-1)^{\ell} & 0 & 0 & 0 \\
0 & & & \\
0 & & A \\
0 & &
\end{array}\right)
$$

telles que $(-1)^{\ell} \mathrm{A} \in \mathrm{SO}_{3}$.

Soient $E$ un espace vectoriel sur $C, Q$ une forme quadratique non dégénérée sur $E$. On sait que le groupe orthogonal spécial $S O(Q)$ n'est pas simplement connexe. Le revêtement universel de $S O(Q)$ est le groupe de Clifford spécial $C^{+}(Q)$ qui est un revêtement de degré 2 de $S O(Q)$. $\pi_{Q}$ désignera la projection de $C^{+}(Q)$ sur $S O(Q)$. Pour la définition de $C^{+}(Q)$, voir chapitre 9, Bourbaki [2].

Proposition 3.11. Soient $E$ un espace vectoriel de dimension finie sur un corps $k$ et $Q$ une forme quadratique sur $E$. Soient $E_{1}, E_{2}$ deux sousespaces de $E$ tels que $E_{1}$ et $E_{2}$ soient orthogonaux, la restriction $Q_{1}$ de $Q$ sur $E_{1}$ soit non dégénéré, $E$ soit la somme directe de $E_{1}$ et $E_{2}$. Alors l'inclusion $i$ de $E_{1}$ dans $E$ induit l'homomorphisme canonique de groupes $i^{+}: C^{+}\left(Q_{1}\right) \rightarrow C^{+}(Q)$ et l'homomorphisme de groupes $i^{\prime}: S O\left(Q_{1}\right) \rightarrow S O(Q)$ tels que $\pi_{Q} \circ i^{+}=i^{\prime} \circ \pi_{Q_{1}}$.

L'homomorphisme $i^{\prime}$ est défini par

$$
i^{\prime}(g)=\left(g, \mathrm{id}_{E_{2}}\right) \quad\left(g \in S O\left(Q_{1}\right)\right) .
$$

La proposition résulte de la définition et des propriétés élémentaires de l'algèbre de Clifford (voir $\S 9, \mathrm{n}^{\circ} 3$, Bourbaki [2]).

Appliquons maintenant la proposition: nous poserons

$$
\begin{aligned}
& E=C^{4}, \quad E_{1}=\left\{{ }^{t}\left(x_{0}, x_{1}, x_{2}, x_{3}\right) \in E \mid x_{0}=0\right\}, \\
& E_{2}=\left\{{ }^{t}\left(x_{0}, x_{1}, x_{2}, x_{3}\right) \in E \mid x_{1}=x_{2}=x_{3}=0\right\}, \\
& Q\left(\left(x_{0}, x_{1}, x_{2}, x_{3}\right)\right)=x_{0}^{2}+x_{1}^{2}+x_{2}^{2}+x_{3}^{2} .
\end{aligned}
$$

CorollaIre 3.12. Si l'on identifie $S O\left(Q_{1}\right)$ à son image dans $S O(Q)$, l'image réciproque $\pi_{Q}^{-1}\left(S O\left(Q_{1}\right)\right)$ est connexe.

$\pi_{Q}: \pi_{Q}^{-1}\left(S O\left(Q_{1}\right)\right) \rightarrow S O\left(Q_{1}\right)$ est un revêtement de degré 2. D'autre part, $\pi_{Q_{1}}: C^{+}\left(Q_{1}\right) \rightarrow S O(Q)$ est aussi un revêtement de degré 2 et on a $\pi_{Q}=\pi_{Q}^{-1} \circ i^{+}$. 
Il en résulte $i^{+}$est un isomorphisme de $C^{+}\left(Q_{1}\right)$ sur $\pi_{Q}^{-1}\left(S O\left(Q_{1}\right)\right)$. Puisque $C^{+}\left(Q_{1}\right)$ est connexe, $\pi_{Q}^{-1}\left(S O\left(Q_{1}\right)\right)$ l'est aussi.

On a morphisme de groupes analytiques de $C^{+}(Q)$ dans $G^{a n}$. Puisqu'il s'agit des groupes analytiques simples, ce morphisme est algébrique. Donc $X$ est un espace homogène algébrique de $C^{+}(Q)$. Il en rèsulte que la classification algébrique de l'espace homogène $(G, X)$ est réduite à la détermination de tous les sous-groupes algébriques $H$ de $C^{+}(Q)$ dont les composantes connexes de l'élément neutre sont

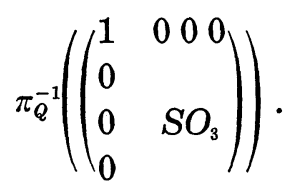

Lemme 3.13. $\pi_{Q}(H)$ a au plus deux composantes connexes. Plus

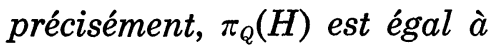

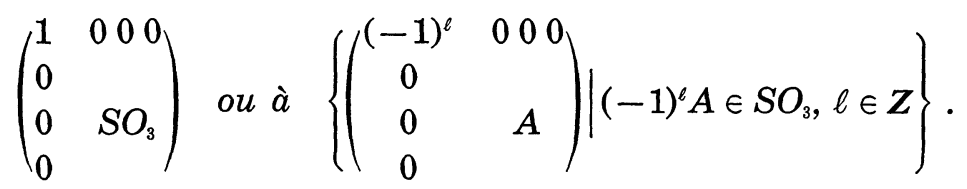

Le raisonnement du lemme 3.7 montre que l'existence d'un élément $\left(b_{i j}\right) \in \pi_{Q}(H)$ tel que le vecteur $\left(b_{12}, b_{13}, b_{14}\right)$ soit différent de zèro, constitue une contradiction. Le lemme en résulte.

D'abord examinons le premier cas

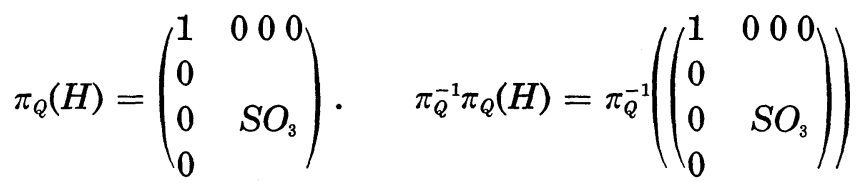

est connexe d'après le corollaire 3.18 , on en conclut

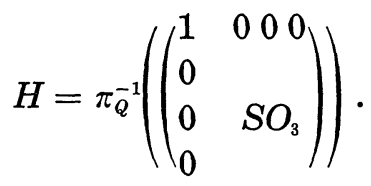

Donc $(G, X)$ est isomorphe en tant qu'opération algébrique à

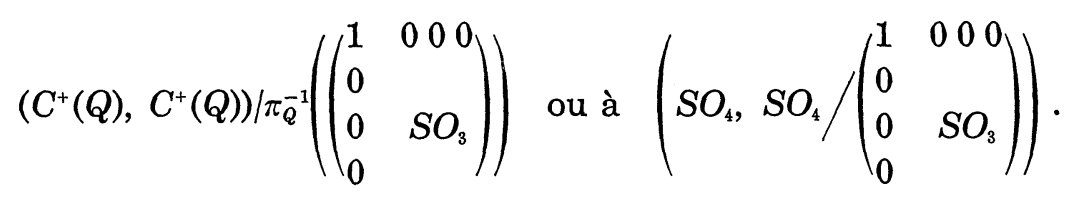


Par hypothèse 2.11, $(G, X)$ est isomorphe à

$$
\left(\mathrm{SO}_{4}, \mathrm{SO}_{4} /\left(\begin{array}{ccc}
1 & 0 & 0 \\
0 & & \\
0 & \mathrm{SO}_{3}
\end{array}\right)\right)
$$

Si $E$ désigne la représentation irréducitible de degré 4 de $\mathrm{SO}_{4}$,

$$
\mathrm{SO}_{4} /\left(\begin{array}{cccc}
1 & 0 & 0 & 0 \\
0 & & \\
0 & \mathrm{SO}_{3} \\
0 &
\end{array}\right)
$$

se réalise comme une orbite de ${ }^{t}(1,0,0,0)$ de $E . \quad(G, X)$ est birationnellement isomorphe au cas (9) du théorème. On suppose maintenant

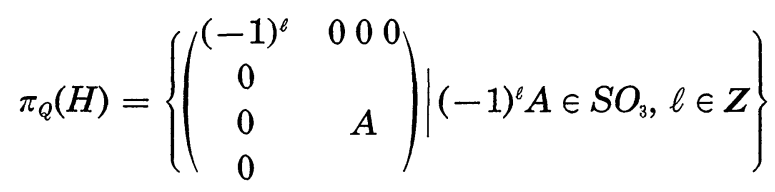

que nous poserons $H^{\prime}$. Il en résulte, comme dans le premier cas, que $(G, X)$ est isomorphe en tant qu'opération algébrique à $\left(C^{+}(Q), C^{+}(Q) / \pi_{Q}^{-1}\left(H^{\prime}\right)\right)$, $\left(\mathrm{SO}_{4}, \mathrm{SO}_{4} / H^{\prime}\right)$ ou $\left(\mathrm{PSO}_{4}, \mathrm{PSO}_{4} / H^{\prime \prime}\right)$ où $H^{\prime \prime}$ est l'image de $H^{\prime}$ par la projection canonique de $\mathrm{SO}_{4}$ sur $\mathrm{PSO}_{4}$. Parmi les trois espaces homogènes, seul le dernier satisfait à la condition 2.11. Donc $(G, X)$ est isomorphe en tant qu'opération algébrique à $\left(\mathrm{PSO}_{4}, \boldsymbol{P}_{3}\right)$, ce qui achève la dèmonstration $\mathrm{du}$ théorème.

La démonstration du théorème montre que la classification analytique globale coïncide avec la classification algébrique globale (dans le cas primitif et $\operatorname{dim} X=3)$. On peut montrer plus généralement: Soient $\left(G_{i}, G_{i} / H_{i}\right)(i=1,2)$ deux opérations algébriques telles que $\left(G_{i}^{a n},\left(G_{i} / H_{i}\right)^{a n}\right)$ soient primitives. Alors deux conditions suivantes sont équivalentes. (1) $\left(G_{1}^{a n},\left(G_{1} / H_{1}\right)^{a n}\right)$ est isomorphe à $\left(G_{2},\left(G_{2} / H_{2}\right)^{a n}\right)$ en tant qu'opérations analytiques. (2) $\left(G_{1}, G_{1} / H_{1}\right)$ est isomorphe à $\left(G_{2}, G_{2} / H_{2}\right)$ en tant qu'opérations algébriques. Voir [15].

Le théorème 3.4 permet de classifier à conjugaison près les sous-groupes algébriques primitifs de $\mathrm{Cr}_{3}$.

CoRollaIRE 3.14. Tout sous-groupe algébrique connexe primitifs de $\mathrm{Cr}_{3}$ est, à conjugaison près, un sous-groupe de $\left(P G L_{4}, P^{4}\right)$ ou $\left(P S O_{5},\left\{x_{0}^{2}+x_{1}^{2}+\right.\right.$ 
$\left.\left.x_{2}^{2}+x_{3}^{2}+x_{4}^{2}=0\right\} \subset P_{4}\right) . \quad$ En particulier, les opération algébriques $\left(P G L_{4}, \boldsymbol{P}_{3}\right)$ et $\left(\mathrm{PSO}_{4},\left\{x_{0}^{2}+x_{1}^{2}+x_{2}^{2}+x_{3}^{2}+x_{4}^{2}=0\right\} \subset \boldsymbol{P}_{4}\right)$ determinent des classes de conjugaison maximales de sous-groupes algébriques connexes de $\mathrm{Cr}_{3}$.

En effet, les opérations (2). (3), (4). (5), (6), (7) du théorème 3.4 sont des sous-opérations de $\left(P G L_{4}, \boldsymbol{P}^{3}\right)$. L'opération (9) est une sous-opération de $\left(P S O_{5},\left\{x_{0}^{2}+x_{1}^{2}+x_{2}^{2}+x_{3}^{2}+x_{4}^{2}=0\right\} \subset P_{4}\right)$. La dernière assertion resulte du fait qu'une opération algébrique primitive n'est jamais une sous-opération d'une opération algébrique imprimitive.

Remarques 3.15.1. On peut montrer la maximalité des classes de conjugaison de sous-groupes algébriques definies par $\left(P G L_{4}, \boldsymbol{P}_{3}\right)$ et $\left(P S O_{5}\right.$, $\left.\left\{x_{0}^{2}+x_{1}^{2}+x_{2}^{2}+x_{3}^{2}+x_{4}^{2}=0\right\}=X \subset \boldsymbol{P}_{4}\right)$ directement. Il est facile de voir que Aut $\boldsymbol{P}_{3}=P G L_{4}$ et Aut $X=P S O_{5}$. Alors la maximalité resulte des théorème 2.9 , théorème 2.10 et de la projectivité des espaces homogènes.

3.15.2. Le raisonnement de 3.15.1 montre: Soient $G$ un groupe algébrique semi-simple et $P$ un sous-groupe parabolique de $G$. Soit $n$ la dimension de la variété rationnelle $G / P$. On désigne par Aut ${ }^{0} G / P$ la composante connexe de l'élément neutre de groupe algébrique Aut $G / P$. Alors (Aut ${ }^{0} G / P, G / P$ ) determine une classe de conjugaison maximale de sousgroupes algébriques connexes de $\mathrm{Cr}_{n}$.

3.15.3. Le théorème de Borel-Weil nous permet de determiner Aut ${ }^{0} G / P$. Signalons qu'il y a des cas où $\operatorname{Aut}^{0} G / P \nsupseteq \nsupseteq G$, par exemple l'opération (4) du théorème 3.4 .

\section{BibliographIE}

[1] Borel, A., Linear algebraic groups, Benjamin, New York 1969.

[2] Bourbaki, N., Algèbre, Chapitre 9, Formes sesquilinéaires et formes quadratiques, Hermann, Paris 1959.

[ 3 ] —-, Groupes et algèbres de Lie, Chapitre 3, groupes de Lie, Hermann, Paris 1972.

[4] Demazure, M., Sous-groupes algébrique de rang maximum du groupe de Cremona. Ann. Scient. Ec. Norm. Sup., 4e série, t. 3 (1970), 507-588.

[5] Enriques, F., Sui gruppi continui di trasformazioni cremoniane nel piano, Rendic. Accad. dei Lincei, 1893, 468-473.

[6] Enriques, F. e Fano, G., Sui gruppi di trasformazioni cremoniane dello spazio, Annali di Matematica pura ed applicata, s. $2^{\mathrm{a}}$, to. 15 (1897), 59-98.

[ 7 ] Fano, G., I gruppi continui primitivi di trasformazioni cremoniane dello spazio, Atti R. Acc. di Torino, 33 (1898), 221-271.

[8] Godeaux, L., Les transformations birationnelles du plan, Mémorial des Sciences Mathématiques, $n^{\circ}$ 122, Gauthier-Villars, Paris 1953. 
[9] Lie, S., Théorie der Transformationsgruppen, dritter und letzter Abschnitt, Teubner, Leipzig 1893.

[10] Mumford, D., Mathematical developments arising from Hilbert problems, Proceeding of symposia in pure mathematics, 28 (1974), 44-45.

[11] Rosenlight, M., Some basic theorems on algebraic groups, Amer. J. of Math., 78 (1956) , 401-443.

[12] Serre, J. P., Algèbre de Lie semi-simples complexes, Benjamin, New-York 1966.

[13] Well, A., On algebraic groups of transformations, Amer. J. Math., 77 (1955), $355-391$.

[14] Umemura, H., Maximal algebraic subgroups of the Cremona group of three variables-imprimitive algebraic subgroups of exceptional type, preprint.

[15] Umemura, H., Un article sur le groupe de Cremona, en préparation.

Nagoya University 